\title{
Metal Recovery Using Polyphenols Prepared by Enzymatic Reactions of Horseradish Peroxidase
}

\author{
Hidetaka Kawakita
}

Department of Applied Chemistry, Saga University, Saga, 840-8502, Japan

\begin{abstract}
Enzymatic reactions of horseradish peroxidase were used to polymerize phenols in various substrates, and the polyphenols were used for precious metal recovery. The phenol groups in polyphenols can reduce gold ions to gold particles at high capacities. Increasing the phenol group density in the polyphenol enhances the gold-reducing ability in acidic media. Poly(tyramine) produced by polymerization with horseradish peroxidase has oxidation and ion-exchange abilities and good solubility. Palladium and platinum ions were adsorbed on poly(tyramine) via ion-exchange interactions. Using the solubility of poly(tyramine), we propose a novel recovery system for gold ions. Because of the hydroxyl groups in the polymer, base metal ions, such as copper ions, were adsorbed in the $\mathrm{pH}$ region of 2-5.
\end{abstract}

Keywords Polyphenol, Horseradish Peroxidase, Metal Recovery

\section{Introduction}

Precious metals are very expensive, and techniques for recycling or refining such metals are needed. In Japan, there is a particular focus on 'urban mines'; electronic and electrical devices containing precious metals are immersed in high-concentration acidic solutions, and the precious metals are leached out from the devices. The leached metal ions are captured for recovery using hydrometallurgical engineering. Coagulation methods, solvent extraction methods, and adsorption methods are used. Adsorption is a classical method for metal recovery, and involves removal of the metals from metal-containing solutions using solids containing ligands. The metal ions move to the ligands in the solid. In adsorption, the design of an adsorbent with high capacity, high selectivity, high speed, and the potential for repeated use is important.

In this study, metal recovery by adsorption with polyphenol compounds is described. Polyphenols occur naturally in fruits and tea leaves. Our group has researched metal recovery using biomass waste containing polyphenols[1-3]. Polyphenols contain electron-donating phenol rings. When gold ions interact with polyphenol-containing biomass wastes in acidic media, the gold ions are reduced to elemental gold, and the gold crystallizes to form gold crystals, observable by the naked eye. Amino groups are easily chemically introduced into biomass waste for the selective recovery of palladium and platinum via ionic interactions in

* Corresponding author:

kawakita@cc.saga-u.ac.jp (HidetakaKawakita)

Published online at http://journal.sapub.org/scit

Copyright (C) 2012 Scientific \& Academic Publishing. All Rights Reserved acidic media. However, biomass wastes have very complicated structures, and contain many biomacromolecules such as lignin, cellulose, and hemicellulose along with polyphenols. If the structure of the polyphenol-containing adsorbent is artificially designed, smarter polyphenol-containing adsorbents can be obtained.

Enzymes are substrate-specific biocatalysts. Enzymatic reactions could be used to produce the polyphenols described above. Horseradish peroxidase (HRP) has high substratespecificity for phenol and aniline structures. Kobayashi and Uyama have already investigated polymerization using HRP[4-6]. Kobayashi's group used phenol and aniline compounds with different structures as substrates to determine the molecular distribution and retained functional group density in the obtained polymers.

Based on Kobayashi's research, we designed polyphenols targeted at metal recovery, especially precious metals. Various phenol compounds were polymerized by HRP reactions for the recovery of gold ions. Tyramine, which contains phenol and amino groups, was polymerized for use in gold, palladium, and platinum recovery. A gold ion recovery system, with recycling of the polymer, is proposed.

\section{Selective Recovery of Gold Ions by Polyphenols}

Polyphenols in fruits and leaves reduce gold ions to elemental gold, forming gold particles via crystallization and aggregation. In this process, the reducing power of the polyphenol is the key property. A high density of the polyphenol in the adsorbent enhances the reducing ability of the polyphenol. Phenols were polymerized by HRP reactions 
using various substrates, as shown in Figure 1[7]. In the substrates, the numbers of hydroxyl groups and alkyl groups in the benzene rings were changed to produce several kinds of polyphenols.
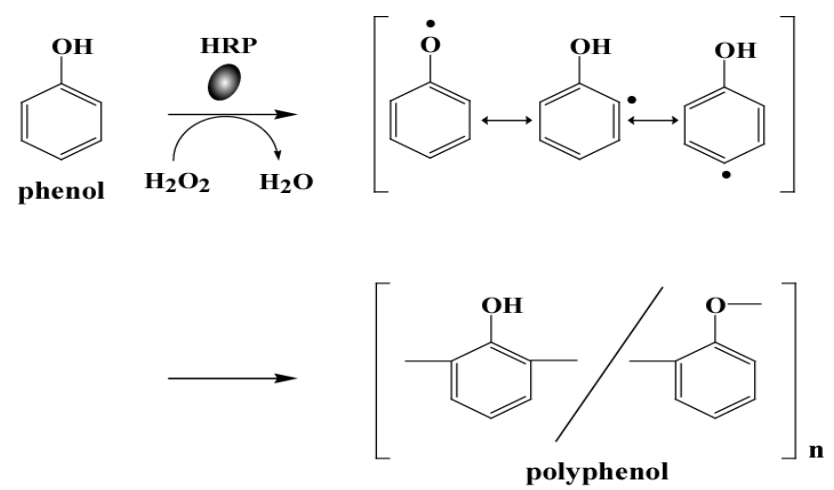

Figure 1. Phenolpolymerization using horseradish peroxidase

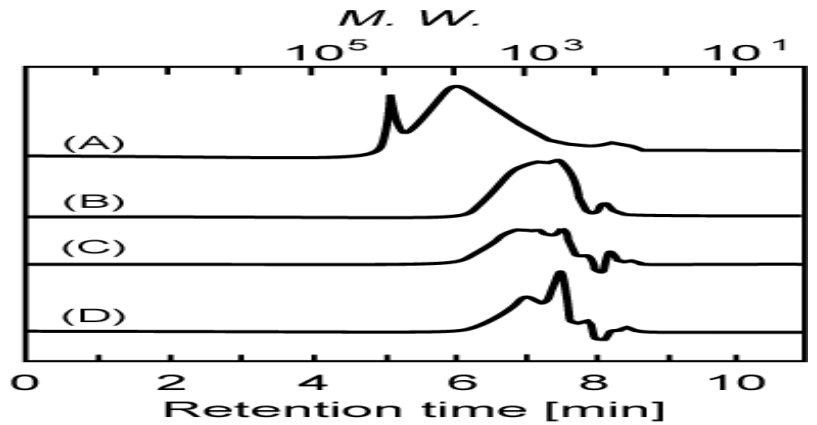

Figure 2. Gel-permeation chromatograms of (A) poly( $m$-cresol), (B) poly (3-methylcatechol), (C) poly (4-methylcatechol), and (D) poly (4ethylcatechol)

The polymerization scheme using the HRP reaction is shown in Scheme 1. The phenol group was recognized as the active site for HRP; iron coordinated with the amino acids at the active site and the peroxides added generated radicals, transferring to the hydroxyl group in the phenol. The radicals at the phenol group start the phenol polymerization. The number of hydroxyl groups and the lengths and locations of the alkyl groups in the substrate influence receipt of radicals from the active site, producing different kinds of polyphenols. In this study, a methanol/water solution was used as the reaction medium. Methanol dissolves the substrate, a phenol-like structure, enabling it to polymerize homogeneously. In the polymerization process, the produced polymer becomes insoluble in the medium and precipitates. It is suggested that polymerization is terminated by precipitation.

Gel-permeation chromatograms of the polyphenols obtained from various substrates are shown in Figure 2. Cresol, which has one hydroxyl group, has high solubility in the reaction medium after polymerization, so a higher- molecular-weight polyphenol is obtained using cresol than with other substrates. The maximum molecular weight was about 4000 , and the degree of polymerization was 30 . In the case of ethylcatechol, which has a hydrophobic group, the molecular weight of the polymer was low, suggesting that, as a result of low solubility in the reaction medium, termination occurred sooner.
In Japan, metal ions in waste electronic devices in urban mines are leached using highly concentrated acids. The leached metal ions dissolved in the acids should be captured selectively. In this study, $\mathrm{HCl}$ solution was used as the acid. First we used gold ions as the target ion. The obtained polyphenol was used to treat gold ions along with other metals, namely platinum, palladium, and base metal ions, because wastewaters contain many kinds of metal ions. The relationship between $\mathrm{HCl}$ concentration and percentage recovery of gold ions is shown in Figure 3. Poly (catechol) gave $100 \%$ recovery. In the presence of other ions, poly (catechol)s had high selectivity for gold ions. In the case of poly (ethylcatechol), increasing the concentration of $\mathrm{HCl}$, especially to above $1 \mathrm{M}$, significantly reduced the percentage recovery of gold ions. However, at $\mathrm{HCl}$ concentrations above $1 \mathrm{M}$, poly (catechol) maintained high selectivity for gold ions.
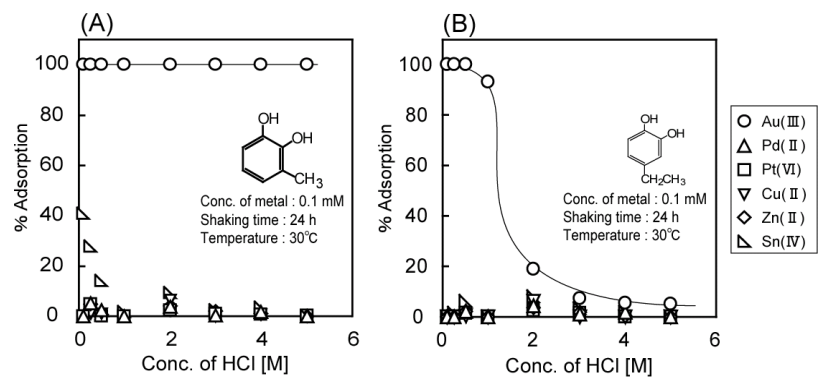

Figure 3. Selective gold-ion recovery using poly (catechol) in $\mathrm{HCl}$ solution

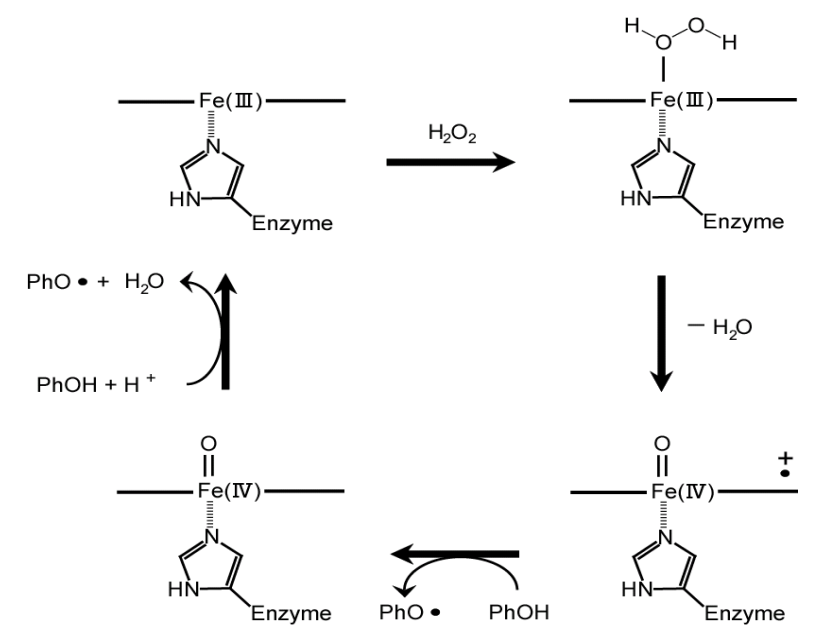

Scheme 1. Radical transfer at the active sites of horseradish peroxidase

Examination of the polyphenol surface showed that gold particles were generated. Gold-particle formation also occurred when fruit waste was used. Gold formation is thought to occur according to the following scheme: 1) electrons are generated from the phenol group, 2) the electrons are transferred to gold ions, 3) gold ions are selectively reduced to form elemental gold as a result of the high oxidation and reduction potentials of gold ions, and 4) the elemental gold forms crystals and aggregates. The reduced percentage of gold recovery by poly (ethylcatechol) with increasing $\mathrm{HCl}$ concentration resulted from non-smooth transfer of electrons generated from the phenol groups, showing that the location 
of the phenol group plays a crucial role in achieving efficient gold-ion reduction.

The reducing abilities of the polyphenols were related to the functional density of the phenol. To improve the reducing ability, pyrogallol, which has three phenol group in its structure, was used as a substrate. In various $\mathrm{HCl}$ solutions, poly (pyrogallol) produced using HRP was used for gold-ion reduction, as shown in Figure 4. At lower gold-ion concentrations, the curve was sharp, demonstrating that gold-ion recovery at low concentrations is possible. The concentration of reduced gold ions was $16 \mathrm{mmol}$ per gram of poly (pyrogallol), a higher recovery rate than those achieved with commercially available materials.

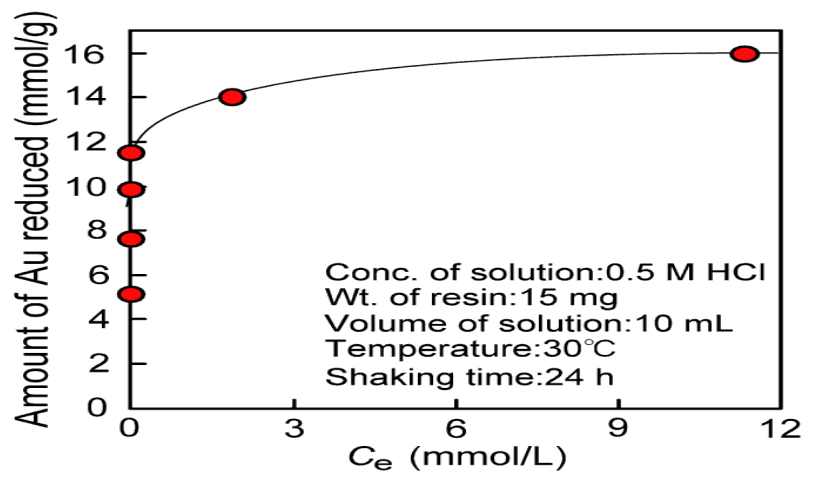

Figure 4. Isotherm curve for gold-ion recovery using poly (pyrogallol)

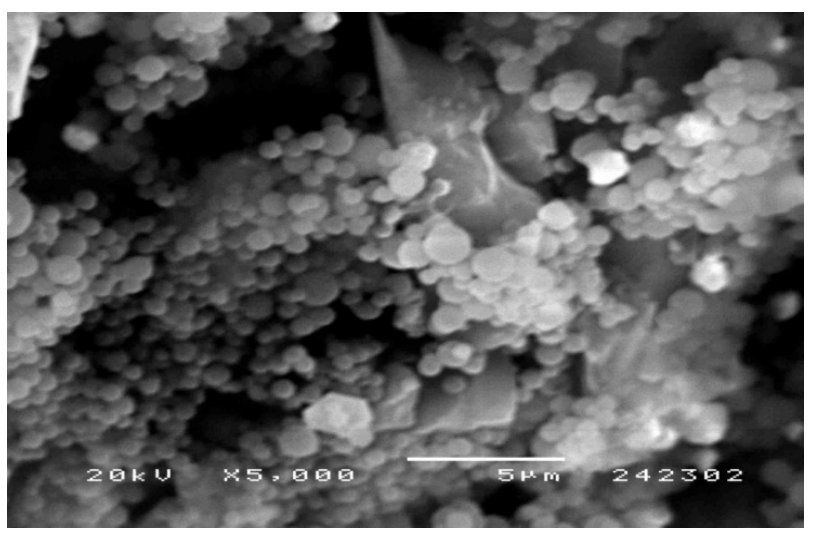

Figure 5. SEM image of polymerized 3-methylcatechol

The morphology of the polyphenol obtained from 3 -methylcatechol is shown in Figure 5. The polyphenol forms spherical shapes via self-assembly, depending on the species in the reaction medium. Catechol, with no alkyl groups, did not form spherical structures, demonstrating that the assembly of hydrophobic groups formed via hydrophobic interactions among 3-methylcatechols is important.

When gold ions were present with phenols, such as catechol, in acidic media, the phenols started to polymerize[8]. This is because catechol donates electrons to the gold ions, forming elemental gold, and the electron-localized phenol compounds are unstable, and interact with neighboring phenols, forming covalent bonds between phenols. The molecular weight of the polymerized polyphenol was about 1000 , and 10 mers. The reducing power, which depended on the number of hydroxyl groups as well as alkyl chain length and location in catechol, was strongly related to the gold-ionreducing power and also to the produced polymer. However, there are no direct relationships between the molecular weight of the polyphenol and the oxidation and reduction potentials of gold ions, suggesting that the polymerization and reduction pathways involve complicated reactions.

\section{Recovery of Gold Ions by Poly (tyramine)}

We found that polyphenols prepared using HRP enzymatic reactions, with high densities of hydroxyl groups, efficiently reduced gold ions to gold particles. The introduction of more complex functions to the polyphenol results in improved precious metal recovery, especially for gold ions[9]. The substrate used for the HRP reactions was tyramine. Tyramine has one phenol group and an amino group in its structure. The tyramine polymerization scheme using HRP is shown in Scheme 2. The phenol group in the obtained polyphenol has the ability to reduce gold ions. As a result of protonation, the amino group in poly (tyramine) can capture the precious metals palladium and platinum via ion-exchange interactions. The amino group affects the polymer solubility by changing the solution conditions such as acid concentration. The alkyl group between the amino group and the benzene ring inhibits electron transfer during polymerization using HRP.
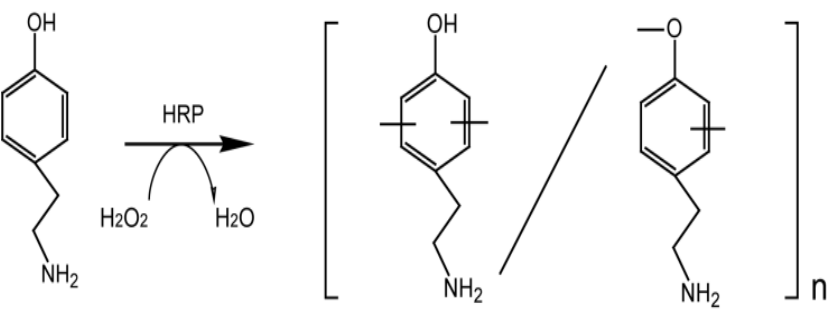

Scheme 2. Polymerization scheme of poly(tyramine)
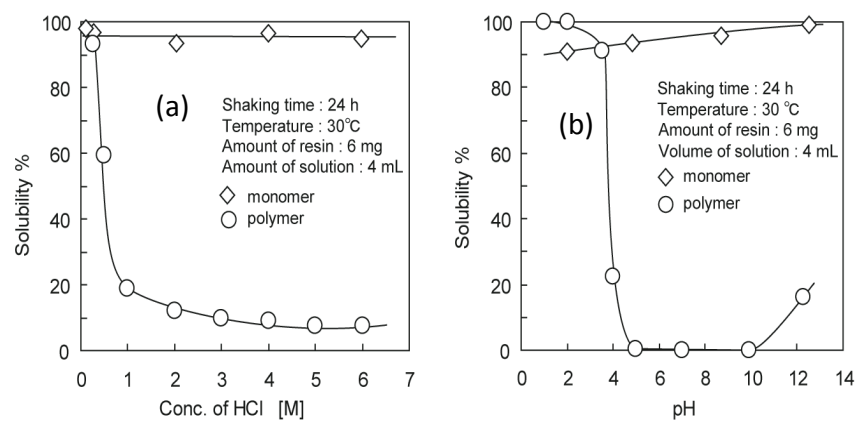

Figure 6. Solubility of poly (tyramine) in (a) $\mathrm{HCl}$ solutions and (b) different $\mathrm{pH}$ regions

The solubilities of poly (tyramine) in various $\mathrm{pH}$ and $\mathrm{HCl}$ concentration regions are shown in Figure 6, along with those of the tyramine monomer. In the case of tyramine, $100 \%$ solubility was obtained. With increasing $\mathrm{HCl}$ concentration, the solubility of poly (tyramine) decreased significantly as a result of the salting-out effect by chloride ions. At lower $\mathrm{HCl}$ 
concentrations and low $\mathrm{pH}$ region, high solubility of poly (tyramine) was observed as a result of protonation of the amino group in the polymer. At $\mathrm{pH} 7.0$, no protonation of the amino group occurred, resulting in no solubility. Above neutral $\mathrm{pH}$, dissociation of the phenol groups started again, solubilizing the polymer. The solution conditions can therefore control the phase changes between dissolved and solid polymer.

Based on the solubility of poly(tyramine), we propose a novel recovery system for gold ions (Figure 7): 1) poly (tyramine) is mixed with gold ions, reducing the ions to gold particles; 2) poly (tyramine) containing reduced the gold particles is immersed in a solution at $\mathrm{pH} 1.0 ; 3$ ) the remaining insoluble gold particles are obtained by filtration; and 4) adjusting the solution to $\mathrm{pH} 7.0$ gives insoluble poly (tyramine) as a precipitate. This system addresses the recycling of poly (tyramine) using a soluble/insoluble polymer by changing the solution conditions. The percentage recoveries of gold for different cycle numbers are shown in Figure 8 ; the results show that the gold-ion-reducing ability of the poly (tyramine) was constant, so poly (tyramine) could be used in smart recycling processes.

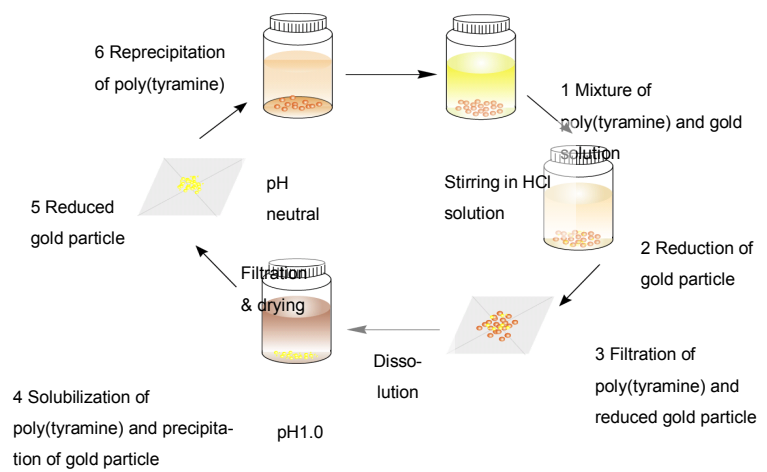

Figure 7. Recycling process for poly(tyramine) for reduction of gold ions

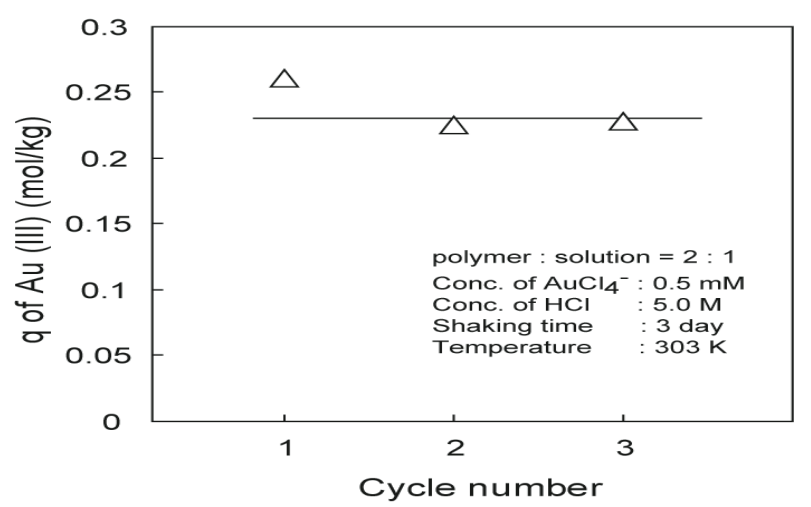

Figure 8. Repeated use of poly(tyramine), according to the scheme in Figure 7

\section{Precious Metal Recovery Using Poly (tyramine)}

Poly(tyramine) is highly soluble in water. However, in solutions with high $\mathrm{HCl}$ concentrations, the polymer be- comes insolublebecause of the salting-out effect. In this case, a solid/liquid heterogeneous system is formed, which can be used for precious metal recovery using hydrometallurgical treatments. When the precious metal ions dissolved in waste electronic devices are the target metals, the solutions are acidic. The leached metal ions in acidic media need to be captured. Because poly (tyramine) has phenol and amino groups, insoluble poly (tyramine) is effective for precious metal recovery. In enzymatic polymerization, only the phenol group is polymerized because of the high selectivity of the substrate. The amino group is retained, without disruption of the structure. The functional group density of amino groups in poly (tyramine) reached $7.3 \mathrm{~mol} / \mathrm{kg}$, so it was expected to have a higher metal-capturing ability than that of commercially available adsorbents.

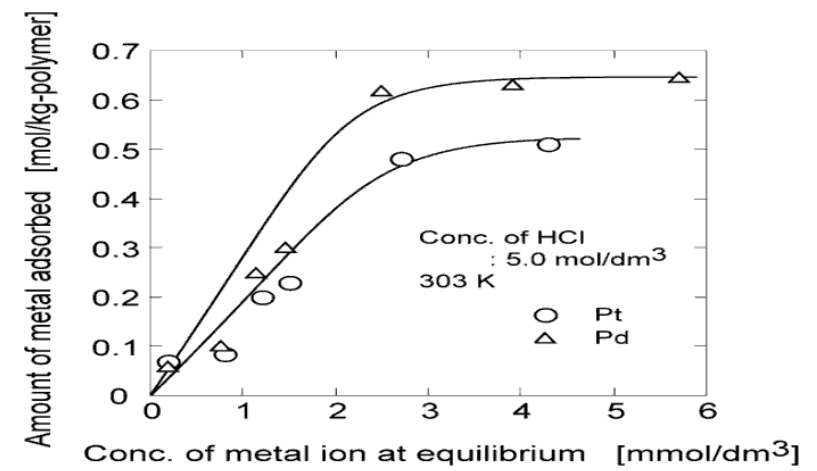

Figure 9. Isotherm curves for palladium and platinum ions adsorption in 5 $\mathrm{M} \mathrm{HCl}$ solution

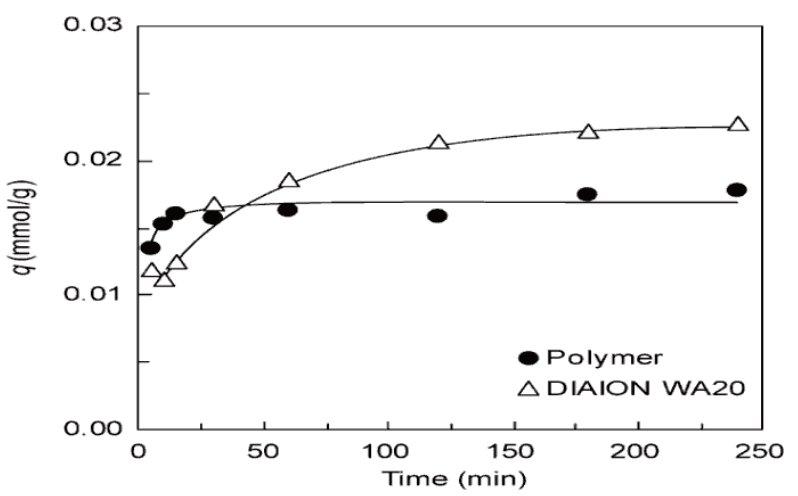

Figure 10. Kinetics of palladium adsorption by poly(tyramine) compared with that of commercially available DIAION WA 20 beads

Palladium and platinum ions dissolved in $5 \mathrm{M} \mathrm{HCl}$ solution were adsorbed on poly(tyramine) at $303 \mathrm{~K}[10]$. The isotherm curve is shown in Figure 9. The level of precious metal absorption achieved was $0.5 \mathrm{~mol} / \mathrm{kg}$. The captured Pd and $\mathrm{Pt}$ ions were not reduced to the elemental forms, unlike the case for gold ions. In $\mathrm{HCl}$ solution, $\mathrm{Pd}$ and $\mathrm{Pt}$ ions form chloride complexes, suggesting that $\mathrm{Pd}$ and $\mathrm{Pt}$ interacted with the amino groups via ionic interactions. The kinetic adsorption of palladium ions, using commercially available beads (DIAION WA 20, Mitsubishi Chemical Corp., Tokyo, Japan) was determined and compared with that of poly (tyramine), as shown in Figure 10. WA 20 has a tertiary amino functional group. In the initial stage, poly(tyramine) gave a higher adsorption performance, but then leveled off. 
This is because poly (tyramine) has a porous structure only on the surface of the adsorbent, so palladium ions smoothly diffuse to the amino group at the surface; however, in the inner part of the adsorbent, poly (tyramine) has a packed structure, making diffusion difficult.

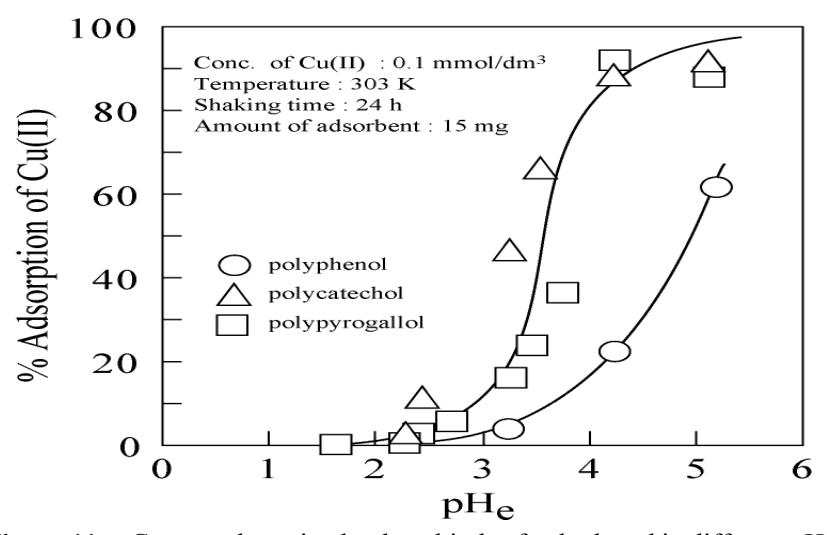

Figure 11. Copper adsorption by three kinds of polyphenol in different $\mathrm{pH}$ regions

\section{Recovery of Other Metal Ions by Polyphenols}

Polyphenols have ion-exchange abilities as well as reducing abilities. The hydroxyl group in the phenol dissociates, forming anions, and dissolved metal cations are captured via ionic interactions[11]. Here, the target metal ion was copper. Three types of polyphenols, prepared from phenol, catechol, and pyrogallol, were produced using the HRP reaction. The $\mathrm{pH}$ dependences of copper adsorption by the three polyphenols are shown in Figure 11. With increasing $\mathrm{pH}$, the percentage of copper ions adsorbed increased. The $\mathrm{pH}$ dependences of adsorption by poly (catechol) and poly (pyrogallol) overlapped, irrespective of the $\mathrm{pH}$, but polyphenol has an affinity for copper capture at higher $\mathrm{pH}$ regions, demonstrating that phenol dissociation and the functional group density of the polyphenol play important roles in ion capture. For divalent copper ions, complexation with inter- or intra-hydroxyl groups in the polyphenol would capture copper ions via ionic interactions.

\section{Summary}

We produced polyphenols, via enzymatic reactions of HRP, for the recovery of precious metal ions, namely gold, palladium, and platinum. The structure of the substrate was changed to obtain various polyphenols. The selective and efficient recovery of gold ions was directly related to the ability of the polyphenol to reduce gold ions to elemental gold particles. The amino groups in poly (tyramine) were used in a novel process for gold recovery via solubilization and insolubilization cycles. Precious metal recovery, especially from urban mines, is crucial for efficient recycling. The polyphenol described here has good potential for use in the recovery of precious metals.

\section{REFERENCES}

[1] H. Kawakita, R. Yamauchi, D. Parajuli, K. Ohto, H. Harada, $\mathrm{K}$. Inoue, "Recovery of gold from hydrochloric acid by means of selective coagulation with persimmon extract," Sep. Sci. Technol., vol. 43, pp. 2375-2385, 2008

[2] D. Parajuli, C. R. Adhikari, H. Kawakita, K. Kajiyama, K. Ohto, K. Inoue, "Reduction and accumulation of $\mathrm{Au}(\mathrm{III})$ by grape waste: A kinetic approach," vol. 68, pp. 1194-1199, 2008

[3] H. Kawakita, M. Abe, J. Inoue, K. Ohto, H. Harada, K. Inoue, "Selective gold recovery using orange waste," Sep. Sci. Technol., vol. 44, pp. 2797-2805, 2009

[4] S. Kobayashi, "Enzymatic polymerization: a new method of polymer synthesis,” J. Polym. Sci. A Polym. Chem., vol. 37, pp. 3041-3056, 1999

[5] T. Oguchi, S. Tawaki, H. Uyama, S. Kobayashi, "Soluble polyphenol," Macromol. Rapid Commun., vol. 20, pp. 401-403, 1999

[6] H. Tonami, H. Uyama, S. Kobayashi, K. Rettig, H. Ritter, "Chemoenzymatic synthesis of a poly(hydroquinone),"Macromol. Chem. Phys., vol. 200, pp. 1998-2002, 1999

[7] H. Kawakita, K. Hamamoto, K. Ohto, K. Inoue, "Polyphenol polymerization by horseradish peroxidase for metal adsorption studies," Ind. Eng. Chem. Res., vol. 48, pp. 4440-4444, 2009

[8] K. Hamamoto, H. Kawakita, K. Ohto, H. Harada, K. Inoue, "Polymerization of phenol derivatives by the reduction of gold ions to gold metal," React. Funct. Polym., vol. 69, 694-697, 2009

[9] H. Kawakita, Y. Yoshimura, K. Ohto, "Reduction of gold ions to gold particles by reusable soluble poly(tyramine) polymerized by horseradish peroxidase," Ind. Eng. Chem. Res., vol. 49, pp. 11582-11586, 2010

[10] Y. Yoshimura, K. Khunathai, A. Nozoe, K. Ohto, H. Kawakita, "Precious metal recovery using poly(tyramine) prepared by radical polymerization with horseradish peroxidase," J. Chem. Eng. Jpn., in press

[11] H. Kawakita, S. Nakano, K. Hamamoto, Y. Matsunaga, Y. Yoshimura, K. Ohto, K. Inoue, "Copper-ion adsorption and gold ion reduction by polyphenols prepared by the enzymatic reaction of horseradish peroxidase," J. Appl. Polym. Sci., vol. 118 , pp. $247-252,2010$ 